\title{
Effects of Annealing Solvents on the Morphology of Block Copolymer-Based Supramolecular Thin Films
}

\author{
Wei-Han Huang, Po-Yu Chen, and Shih-Huang Tung* \\ Institute of Polymer Science and Engineering, National Taiwan University, Taipei, 10617 Taiwan
}

Supporting Information

ABSTRACT: We report a strategy that combines supramolecular assembly and solvent annealing to manipulate the microdomain orientation in block copolymer thin films. In supramolecular thin films formed by polystyrene- $b$-poly(4vinylpyridine) (PS-b-P4VP) with 3-pentadecylphenol (PDP)

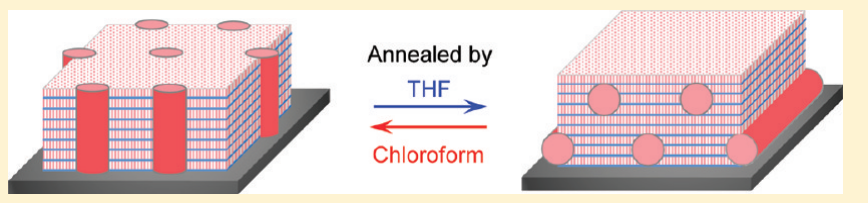
hydrogen-bonded onto P4VP blocks, where PS blocks phaseseparate into cylindrical microdomains, we found that the orientation of PS cylinders can be controlled by using different types of solvents to anneal the films. As films are annealed under the vapors of solvents bearing no oxygen atoms, such as chloroform, PS cylinders are perpendicular to the surface, while under those of solvents bearing oxygen, such as THF, PS cylinders are parallel to the surface. Furthermore, the orientation is switchable upon the alternate use of different types of solvents. The ${ }^{1} \mathrm{H}$ NMR measurements showed that the strengths of hydrogen bonds between PDP and P4VP are greatly weakened in oxygen-bearing solvents due to the competition of the highly electronegative oxygen atoms. We thus suggest that the binding capability of PDP onto P4VP is the key to determine whether the supramolecules can be assembled in the vapors of different types of solvents, which in turn, regulates the orientation of PS cylinders.

\section{INTRODUCTION}

Block copolymers formed by covalently linked incompatible homopolymers phase-separate into a variety of nanoscaled microdomains, typically spherical, cylindrical, lamellar, and gyroidal. ${ }^{1}$ For block copolymers with narrow distribution of molecular weights and under proper thermal treatments, the microdomains can be packed in a highly ordered manner, which is an effective technique for manipulating structures in nanoscale. In bulk samples, however, the orders are limited in grains and the grains are usually joined randomly, such that the overall properties are still isotropic. When block copolymers are fabricated into thin films with nanoscaled thicknesses, the effects of two additional interactions become significant, namely the polymer-substrate and polymer-air interactions. ${ }^{2}$ The phase that interacts most strongly with the substrate prefers to stick on the substrate while the phase with the lowest surface tension tends to expose to air. Both forces regulate the alignments of the microdomains and when the thicknesses of films are thinner than the influential range of the interactions, the microdomains in the entire films will be forced to be oriented along the direction of thickness. In most cases, the cylindrical or lamellar microdomains prefer to be parallel to the surface.

The search of the approaches to obtain perpendicular microdomains in thin films has been an emerging topic recently due to its scientific merit and application potency. ${ }^{3-7}$ To do so, the above-mentioned two interactions need be eliminated or overcome, which has been achieved by surface modifications, ${ }^{3,8-15}$ external stimuli, ${ }^{16,17}$ and nonequilibrium approaches. ${ }^{18-21}$ Another strategy that has been proven to be efficient is the supramolecular assembly. ${ }^{22-27}$ The supra- molecules are formed by block copolymers with small molecules associated with one of the blocks via weak secondary bonds. ${ }^{28-31}$ One major effect of the small molecules is that they alter the relative volume fraction of each component, therefore causing a morphological transition and creating a variety of hierarchical structures. In addition, it has been shown that there is a strong correlation between the microdomain orientation in thin films and the content of the associated small molecules. ${ }^{23-26}$ In general, at sufficiently high ratio of small molecules, the orientation is driven to be perpendicular. This is a simple approach to tailor the microdomain orientation in block copolymer thin films since the amount of weakly bonded small molecules can be adjusted by physical means rather than chemical reaction.

Solvent annealing is usually adopted to replace thermal annealing for processing supramolecular thin films since small molecules may evaporate at high temperature and a tiny amount loss of small molecules would result in a great structural change in such thin films. ${ }^{24}$ Different from thermal annealing, solvent molecules are extra components introduced into films and the final morphology may thus be affected by the interplays between solvents and polymers, which on one hand complicates the structural analysis and on the other hand provides opportunities to create structures that cannot be achieved by thermal annealing. ${ }^{32}$ It has been shown that the type of solvents used for annealing plays an important role in the morphology of block copolymer thin films. ${ }^{33-35}$ For

Received: October 31, 2011

Revised: January 1, 2012

Published: January 18, 2012 
supramolecular thin films, the effects of annealing solvents on the morphology of polystyrene- $b$-poly(4-vinylpyridine) (PS- $b$ P4VP) with 2-(4'-hydroxybenzeneazo) benzoic acid (HABA) molecules have been reported by Minko and co-workers. ${ }^{22,36,37}$ They found that as films were annealed by chloroform, the P4VP(HABA) cylindrical microdomains were parallel to the surface, while annealed by 1,4-dioxane, the cylinders were perpendicular to the surface, which was attributed to the different selectivity of the solvents to each phase. The previous discussions of block copolymer-based supramolecules have been generally under the presumption that the small molecules are firmly grafted on the polymer chains. However, it should be aware that the lifetimes of secondary weak bonds are much shorter than that of covalent bonds and the strength of weak interactions is subject to the influence of environments. It has been shown that hydrogen bonds, through which most supramolecules are formed, would be weakened in the presence of additional molecules with strong electronegative atoms, such as oxygen and nitrogen. ${ }^{38,39}$ In other words, the solvents that diffuse into thin films may interfere with the original interactions in supramolecules.

For the widely studied supramolecular thin films formed by PS- $b$-P4VP with hydrogen-bonded small molecule 3-pentadecylphenol (PDP), as shown in Figure 1, the structures were

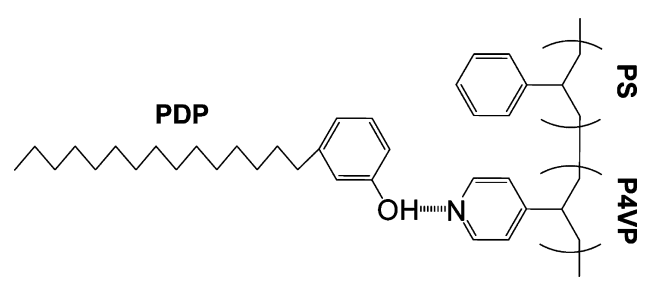

Figure 1. Chemical structure of PS-b-P4VP(PDP) supramolecules.

usually characterized after annealing in the vapor of chloroform. $^{24-26,40-42}$ In this paper, we systematically investigated the effects of annealing solvents on the morphology of PS- $b$ P4VP(PDP) supramolecular thin films. We divide the solvents into two groups: one group contains no oxygen atom on their chemical structures, i.e., nonoxygen-bearing solvents, and the other group contains oxygen atoms, i.e., oxygen-bearing solvents. The oxygen atom is highly electronegative and capable of forming hydrogen bonds. The solvents and their properties are listed in Table 1. The classification is based on the fact that the morphologies of supramolecular thin films annealed by the solvents in each group are similar. However, nonoxygen-bearing solvents cause the PS cylindrical micro- domains oriented perpendicularly while oxygen-bearing solvents lead to parallel alignment. Furthermore, the orientation is switchable when films are alternately annealed by the solvents in different groups. We provide a mechanism originated from the strengths of hydrogen bonds in the presence of different types of solvents to explain why this facile approach can be used to control the orientation of block copolymer thin films.

\section{EXPERIMENTAL SECTION}

Materials. PS- $b$-P4VP with $M_{\mathrm{n}, \mathrm{PS}}=20000, M_{\mathrm{n}, \mathrm{P} 4 \mathrm{VP}}=17000 \mathrm{~g} / \mathrm{mol}$, and PDI $=1.08$ was purchased from Polymer Source Inc. P4VP homopolymer with $M_{\mathrm{w}}=60000 \mathrm{~g} / \mathrm{mol}$ and 3- $n$-pentadecylphenol (PDP) (95\% purity) were purchased from Sigma-Aldrich. Chloroform, benzene and $n$-hexane were purchased from Sigma-Aldrich; toluene, tetrahydrofuran (THF) and N,N-dimethylformamide (DMF) were purchased from Mallinckrodt Chemicals; methanol and ethanol were purchased from ECHO chemicals; 1,4-dioxane was purchased from J.T. Baker. All the deuterated solvents used for ${ }^{1} \mathrm{H}$ NMR measurements were purchased from Sigma-Aldrich. All chemicals were used as received.

Sample Preparation. PS- $b$-P4VP was first dissolved in chloroform to form $1-2 \%(\mathrm{w} / \mathrm{v})$ stock solutions. PDP in a 1:1 molar ratio to $4 \mathrm{VP}$ unit was also dissolved in chloroform. The PS- $b$-P4VP solution was then added dropwise to the PDP solution, followed by stirring for at least 1 week to ensure PDP molecules are hydrogen-bonded with P4VP. Thin films were prepared by spin-coating the mixed solutions onto silicon wafers at spinning speed $3000 \mathrm{rpm}$. Sample thicknesses were measured using a Filmetrics F20 interferometer. For solvent annealing, thin films were placed together with a beaker of $40 \mathrm{~mL}$ solvents inside an inverted dish $(170$ Diam $\times 90 \mathrm{H} \mathrm{mm})$ on a hot plate which heated up the solvents to $30^{\circ} \mathrm{C}$. Thin films were annealed for $48 \mathrm{~h}$. After annealing, the inverted dish was immediately removed and solvents were allowed to freely evaporate.

AFM and TEM Imaging. Atomic force microscopy (AFM) was performed on a MultiMode AFM system with a Nanoscope 3D controller (Digital Instruments) in tapping mode. The spring constant of the cantilever (Nanosensor PPP-NCHR) was $\sim 42 \mathrm{~N} / \mathrm{m}$ and the resonant frequency was $\sim 330 \mathrm{kHz}$. To prepare thin film samples for cross-sectional transmission electron microscope (TEM) imaging, a thin layer of platinum was sputtered onto the surfaces of thin films and then the films were attached to epoxy resin and cured at room temperature for 2 weeks. The substrates were peeled off from the embedded films after the cured epoxy cubes were dipped into liquid nitrogen. The embedded films were then microtomed at room temperature. The thin sections, $\sim 80 \mathrm{~nm}$ in thickness, were exposed to iodine vapor for $2 \mathrm{~h}$ that selectively stains the P4VP blocks to enhance the contrast. TEM images were collected on a Joel JEM-1230 transmission electron microscope at an accelerating voltage of $100 \mathrm{kV}$.

GISAXS. Grazing incidence small-angle X-ray scattering (GISAXS) was conducted on beamline BL23A1 in the National Synchrotron Radiation Research Center (NSRRC), Taiwan. ${ }^{43}$ A monochromatic

Table 1. Properties of Solvents and Resulting Thin Film Structures ${ }^{a}$

\begin{tabular}{|c|c|c|c|c|c|c|}
\hline & \multicolumn{3}{|c|}{ Nonoxygen-bearing } & \multicolumn{3}{|c|}{ Oxygen-bearing } \\
\hline & Toluene & Benzene & Chloroform & THF & 1,4-Dioxane & DMF \\
\hline Solubility parameter $\left(\mathrm{MPa}^{1 / 2}\right)^{44}$ & 18.2 & 18.8 & 19.0 & 18.6 & 20.5 & 24.8 \\
\hline Boiling point $\left({ }^{\circ} \mathrm{C}\right)^{44}$ & 111 & 80 & 61 & 66 & 102 & 153 \\
\hline Soluble components & PS, PDP & PS, PDP & PS, P4VP, PDP & PS, PDP & PS, PDP & P4VP, PDP \\
\hline Orientation of PS cylinders & $\perp$ & $\perp$ & $\perp$ & $\|$ & $\|$ & $\|$ \\
\hline Spacing of PS cylinders (nm) & 35.3 & 35.3 & 35.7 & 33.6 & 34.7 & 32.1 \\
\hline Spacing of P4VP(PDP) lamellae (nm) & 3.9 & 3.9 & 3.7 & 3.8 & 3.7 & 3.9 \\
\hline
\end{tabular}

${ }^{a}$ Key: $(\|)$ parallel to the surface; $(\perp)$ perpendicular to the surface. See ref 44 for solubility and BP data. 

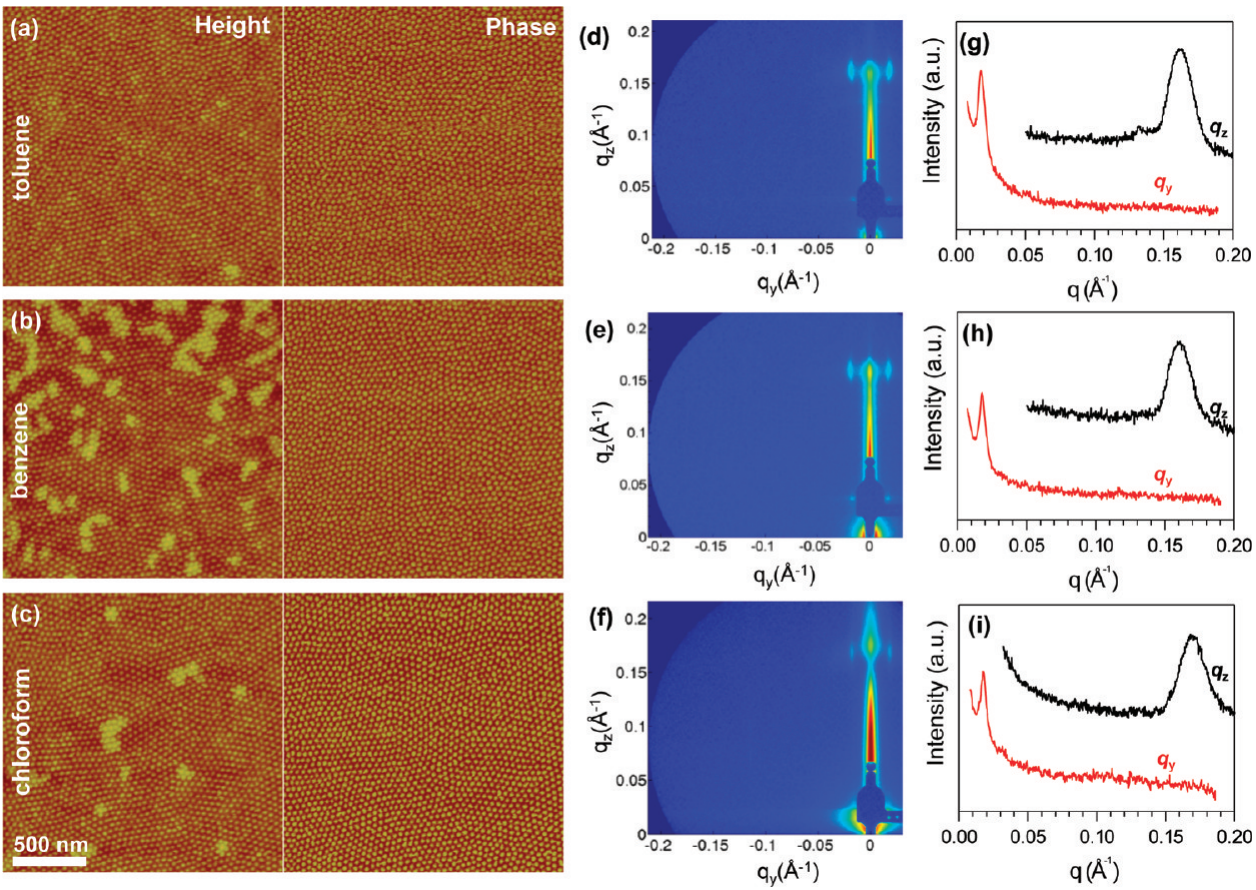

Figure 2. Structure characterization of thin films annealed by nonoxygen-bearing solvents: $(a-c)$ AFM images, (d-f) 2-D GISAXS patterns, and (gi) $q_{y}$ and $q_{z}$ scans from $2 \mathrm{D}$-GISAXS patterns. (a, d, g) a $\sim 41 \mathrm{~nm}$ thin film annealed by toluene, (b, e, h) a $\sim 42 \mathrm{~nm}$ thin film annealed by benzene, (c, f, i) a $\sim 50 \mathrm{~nm}$ thin film annealed by chloroform. The $q_{y}$ scans were extracted at $q_{z}=0.160 \AA^{-1}$ and the $q_{z}$ scans were extracted at $q_{y}=0.018 \AA^{-1}$.

beam of $\lambda=1.240 \AA$ was used and the incident angle was $0.2^{\circ}$. The scattering patterns were collected on a Mar-CCD with a diameter of $165 \mathrm{~mm}$. The scattering intensity profiles were reported as the plots of the scattering intensity $I$ vs the scattering vector $q$, where $q=(4 \pi / \lambda)$ $\sin (\theta / 2)$ and $\theta$ is the scattering angle.

${ }^{1} \mathrm{H}$ NMR. Samples for ${ }^{1} \mathrm{H}$ NMR measurements were prepared by dissolving PDP, PS- $b$-P4VP, and PS- $b$-P4VP(PDP) in dueterated solvents, respectively, at a concentration $\sim 0.25 \%$ (wt $/ v)$. The measurements were conducted on a Bruker DPX $400 \mathrm{MHz}$ system.

\section{RESULTS AND DISCUSSION}

PS- $b$-P4VP which contains approximately symmetric blocks phase-separates into lamellar structures and when spun-cast into thin films on silicon substrate, the surface morphology probed by AFM is featureless due to the parallel lamellae driven by the preferential interaction between P4VP blocks and substrate and the lower surface tension of PS blocks that reduces the surface free energy at film-air interfaces. Upon the addition of PDP in a molar ratio of 1.0 to $4 \mathrm{VP}$ unit, the weight fraction of $\mathrm{P} 4 \mathrm{VP}(\mathrm{PDP})$ complex in the supramolecules, denoted as PS- $b$-P4VP(PDP), is 76.8 wt \%. For such supramolecules, PS blocks form cylinders embedded in P4VP(PDP) matrix where P4VP(PDP) complexes stack into small lamellae with a spacing $\sim 4 \mathrm{~nm}$, i.e., a cylinder-withinlamella hierarchical structure, as reported in our previous paper. $^{26}$

Morphology Caused by Nonoxygen-Bearing Solvents. We first present the thin film morphologies of the supramolecules annealed by nonoxygen-bearing solvents, whose solubility parameters are usually low, as listed in Table 1. Figure $2 \mathrm{a}-\mathrm{c}$ shows the AFM images of thin films, $40-50 \mathrm{~nm}$ in thickness, annealed in toluene, benzene, and chloroform vapors, respectively. The surface morphologies of the three samples are similar, all showing hexagonally packed dots. Such patterns imply that PS cylinders are oriented perpendicular to the surface and the dots are the cross sections of PS cylinders.
To further verify the structures inside the films, the crosssectional TEM image of a representative film, $\sim 65 \mathrm{~nm}$ in thickness, annealed by chloroform was taken, as shown in Figure 3a. The deep black layer is the sputtered platinum and
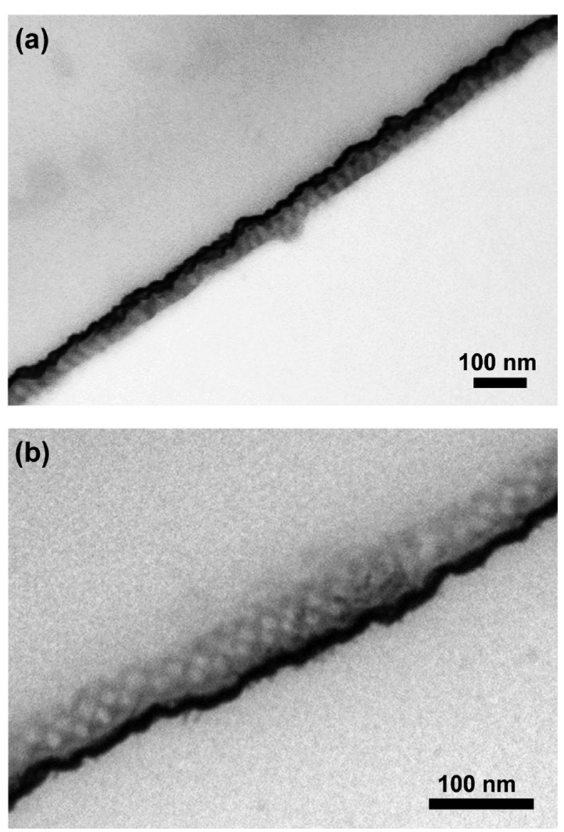

Figure 3. Cross-sectional TEM images of $\sim 65 \mathrm{~nm}$ thin films annealed by (a) chloroform, a nonoxygen-bearing solvent, and (b) THF, an oxygen-bearing solvent.

the original film-air interface is next to the platinum. The darker regions are $\mathrm{P} 4 \mathrm{VP}(\mathrm{PDP})$ matrix due to the staining of iodine and the brighter pillars across the film are PS cylinders, which confirms the formation of perpendicular cylinders. 

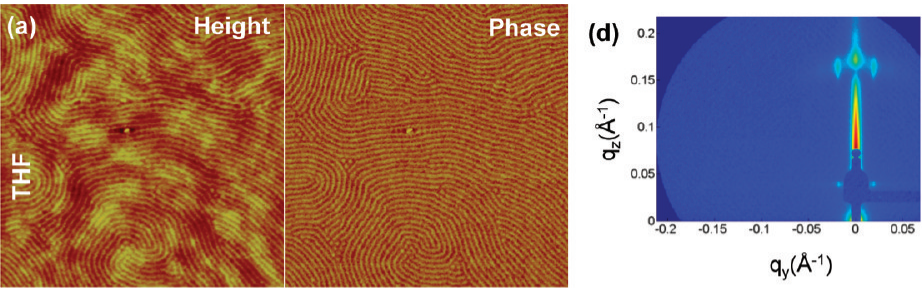

$q_{y}\left(\AA^{-1}\right)$
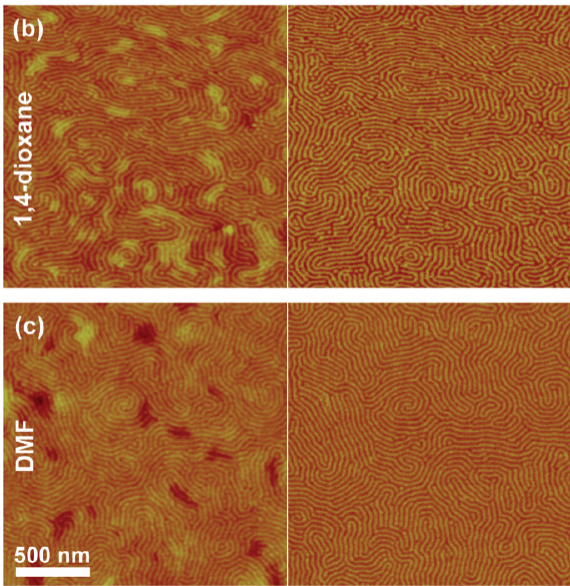

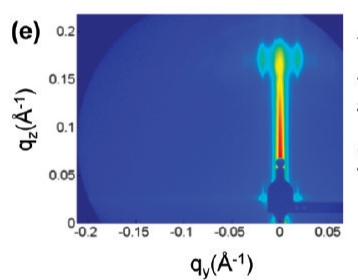

$q_{y}\left(\AA^{-1}\right)$

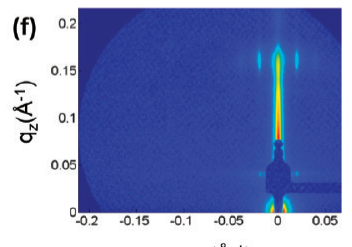

$q_{y}\left(\AA^{-1}\right)$
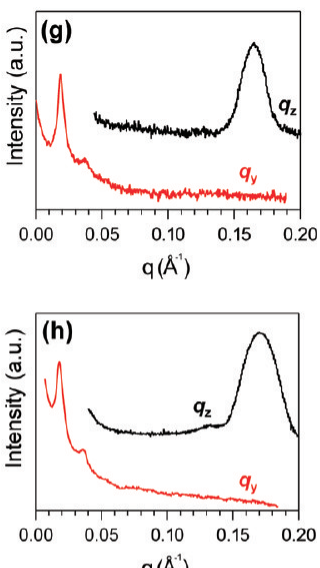

$\mathrm{q}\left(\AA^{-1}\right)$

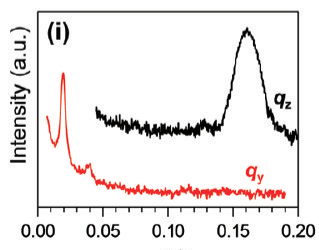

$\mathrm{q}\left(\mathbb{A}^{-1}\right)$

Figure 4. Structure characterization of thin films annealed by oxygen-bearing solvents: $(a-c)$ AFM images, $(\mathrm{d}-\mathrm{f})$ 2-D GISAXS patterns, and ( $\mathrm{g}-\mathrm{i}$ ) $q_{y}$ and $q_{z}$ scans from 2D-GISAXS patterns. (a, d, g) a $\sim 46 \mathrm{~nm}$ thin film annealed by THF, (b, e, h) a $\sim 41 \mathrm{~nm}$ thin film annealed by 1,4-dioxane, (c, f, i) a $\sim 41 \mathrm{~nm}$ thin film annealed by DMF. The $q_{y}$ scans were extracted at $q_{z}=0.160 \AA^{-1}$ and the $q_{z}$ scans were extracted at $q_{y}=0.019 \AA^{-1}$.

The GISAXS data of the three films are displayed in Figure $2 \mathrm{~d}-\mathrm{i}$. All the 2D-patterns (Figure 2d-f) show that diffraction spots appear along both $q_{y}$ and $q_{z}$ axes, and the diffraction spots in $q_{y}$ direction are at lower $q$, i.e., the large-scaled PS cylinders are perpendicular to the surface, while the diffraction spots in $q_{z}$ direction are at higher $q$, i.e., the small-scaled P4VP(PDP) lamellae are parallel to the surface. Note that perpendicular cylinders normally show diffraction rods in $q_{y}$ direction around the baseline slightly above $q_{z}=0 .^{7}$ However, in the present case, the diffraction spots in $q_{y}$ direction at $q_{z} \sim 0.16 \AA^{-1}$ are stronger than those around the baselines at $q_{z} \sim 0.03 \AA^{-1}$. This is because the diffraction beams produced by PS cylinders that are supposed to give bright rods on the baseline now serve as incident beams for small P4VP(PDP) lamellae and the "incident" beams are scattered again by the small lamellae to form the strong diffraction spots at $q_{z} \sim 0.16 \AA^{-1}$. Similar GISAXS patterns have been found in other studies dealing with PS- $b$-P4VP(PDP) system. ${ }^{24-26} q_{y}$ and $q_{z}$ scans retrieved from the 2-D patterns are shown in Figure $2 \mathrm{~g}$-I, where the $q_{y}$ scans were extracted at $q_{z}=0.160 \AA^{-1}$ and the $q_{z}$ scans were extracted at $q_{y}=0.018 \AA^{-1}$. The spacings of PS cylinders for the three solvents estimated from $q_{y}$ scans are in the range of $35-36 \mathrm{~nm}$ and the spacings of small P4VP(PDP) lamellae estimated from $q_{z}$ scans are 3.7-3.9 $\mathrm{nm}$. The detailed data are listed in Table 1 . Note that as shown in the AFM height images, particularly in parts $b$ and $c$ of Figure 2, islands are seen on the surfaces, with a small height $\sim 3-4 \mathrm{~nm}$ close to the spacing of P4VP(PDP) lamellae. Such a terrace topography also confirms that P4VP(PDP) lamellae are oriented parallel to the surface. ${ }^{42}$

By combining the results of AFM, TEM and GISAXS, it is evidenced that in the vapors of nonoxygen-bearing solvents, PS cylinders in PS- $b$-P4VP(PDP) thin films are perpendicular to the surface while $\mathrm{P} 4 \mathrm{VP}(\mathrm{PDP})$ lamellae are parallel to the surface, as illustrated in Figure 5a. It has been known that the orientation of microdomains in block copolymer thin films is dependent on film thickness. If the thickness and the nature period of microdomains are incommensurate, a perpendicular alignment may occur. ${ }^{45}$ In the present case and other studies, $^{24-26}$ a series of thicknesses have been examined and the perpendicular microdomains were consistently observed, indicating that the perpendicular PS cylinders are not caused by the variation of film thickness.

Morphology Caused by Oxygen-Bearing Solvents. For thin films annealed in the vapors of oxygen-bearing solvents, the surface patterns are completely different from those annealed by nonoxygen-bearing solvents. Figure $4 a-c$ show the AFM images of PS- $b$-P4VP(PDP) thin films, $40-50 \mathrm{~nm}$ in thickness, annealed by THF, 1,4-dioxane, and DMF, respectively. All the thin films show fingerprint-like patterns, which are typical of cylindrical microdomains parallel to the surface. A representative cross-sectional TEM of a film, $\sim 65$ $\mathrm{nm}$ in thickness, annealed by THF is shown in Figure $3 \mathrm{~b}$, where hexagonally packed dots are clearly seen. The dots are the cross sections of the parallel PS cylinders. On the surfaces shown in the AFM height images (Figure $4 a-c$ ), the formation of islands or holes with heights or depths $\sim 3-4 \mathrm{~nm}$ implies that similar to thin films annealed by nonoxygen-bearing solvents, the small P4VP(PDP) lamellae here are also parallel to the surface.

The GISAXS data of thin films annealed by THF, 1,4dioxane, and DMF are shown in Figure $4 \mathrm{~d}-\mathrm{i}$. In the $2 \mathrm{D}$ patterns (Figure $4 \mathrm{~d}-\mathrm{f}$ ), clear diffraction spots can be seen in $q_{z}$ direction between $0.16-0.17 \AA^{-1}$, which confirms the P4VP(PDP) lamellae with a spacing $\sim 3.8 \mathrm{~nm}$ are parallel to the surface. Diffraction spots also appear in $q_{y}$ direction at low $q$ and interestingly, two distinct peaks with relative position 1:2 can be observed from the $q_{y}$ scans shown in Figure $4 \mathrm{~g}-\mathrm{i}$, which were extracted at $q_{z}=0.160 \AA^{-1}$. Such a sequence of peak positions is usually interpreted as lamellar structures but it is not the case here since TEM has demonstrated that PS phases form cylinders. It has been shown that when hexagonally 
packed cylinders are well oriented parallel to the surface in thin films, the 1:2 relative peak position may be detected in $q_{y}$ axis. ${ }^{46}$ However, since thin films with 40-50 nm thickness contain only $\sim 2$ cylinder layers, it is more likely that the lamellar-like patterns are produced from the individual cylinder layer where the spatial arrangement of the parallel cylinders is analogous to that of perpendicular lamellae. The center-to-center distance of the cylinders are $32-35 \mathrm{~nm}$ estimating from the first-order peaks. The detailed spacings obtained from GISAXS data for the three annealing solvents are listed in Table 1. The proposed thin film structure annealed by oxygen-bearing solvents is schematically illustrated in Figure 5b. In sum, both PS cylinders and P4VP(PDP) lamellae are parallel to the surface.

(a) Non-oxygen-bearing solvents

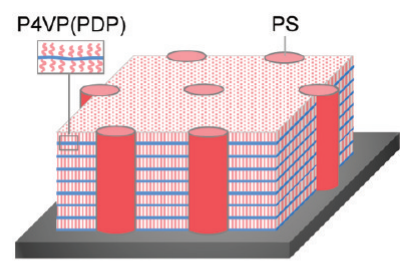

(b) Oxygen-bearing solvents

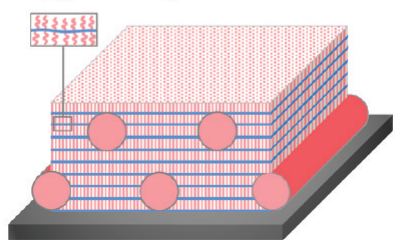

Figure 5. Schematic illustration of proposed structures. (a) Annealed by nonoxygen-bearing solvents, the PS cylinders are perpendicular to the surface while the P4VP(PDP) lamellae are parallel to the surface. (b) Annealed by oxygen-bearing solvents, both the PS cylinders and P4VP(PDP) lamellae are parallel to the surface.

The above results reveal that the orientation of cylindrical microdomains can be tuned by different annealing solvents and we now further show that the orientation is reversible. Here we used chloroform and THF as annealing solvents. As shown in Figure 6 , the morphology of an as-spun film, $\sim 65 \mathrm{~nm}$ in thickness, was a mixture of perpendicular and parallel short cylinders. After annealed by chloroform, perpendicular PS cylinders were formed. We then annealed it again but in the vapor of THF and PS cylinders were driven to be parallel. Oppositely, the cylinders in thin films annealed first in the vapor of THF and subsequently in the vapor of chloroform were transformed from parallel to perpendicular. It is thus demonstrated that the supramolecular assembly is a simple and efficient approach to achieve the repeatable switch of the microdomain orientation in block copolymer thin films, which, as will be discussed later, is due to the reversible nature of supramolecules.

Morphology Caused by Other Solvents. It is apparently that the above solvents can impart mobility to supramolecules in thin films so that the supramolecules can reorganize into more ordered structures after annealing, though the mobility of each component may be in different level. In addition to the above two groups of solvents, we have also used other solvents to anneal the supramolecular thin films, including $n$-hexane, methanol and ethanol. As shown in the Supporting Information, Figure S1, the morphologies annealed by these

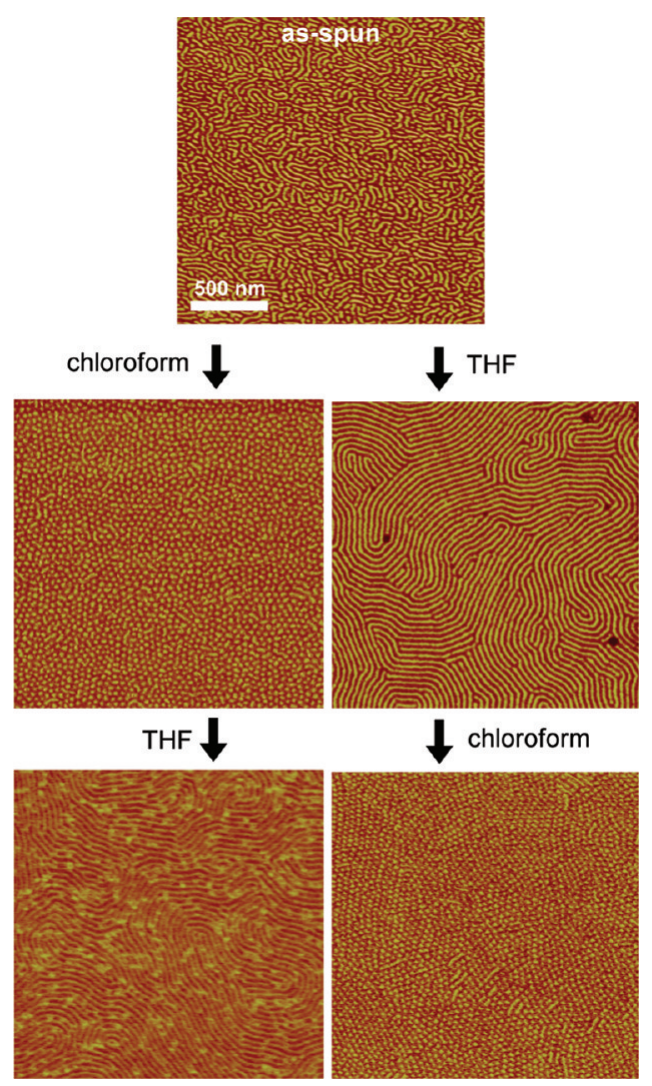

Figure 6. AFM phase images of an as-spun thin films, $\sim 65 \mathrm{~nm}$ in thickness, annealed by chloroform and THF in opposite sequence. The orientation is reversible.

three solvents are nearly identical to those of as-spun films, i.e., still a mixture of parallel and perpendicular cylinders, implying that the supramolecular chains are unable to do long-range motion under annealing. Note that all the three solvents are nonsolvent to PS while P4VP is soluble in methanol and ethanol. Although methanol and ethanol provide mobility to P4VP chains, the diffusion of the whole chains is hindered by the frozen PS chains, thereby no change caused in morphology.

Mechanism. We now discuss the mechanism by which annealing solvents cause parallel or perpendicular orientation of microdomains in supramolecular thin films. We have seen that whether the solvents are composed of oxygen or not, the smallscaled lamellae formed by P4VP(PDP) complexes are always parallel to the surface. At the bottoms of the films, the relatively hydrophilic P4VP blocks preferentially interact with the silicon substrate so that P4VP chains are attracted to the filmsubstrate interfaces and the tails of the associated PDP molecules are repelled from the interfaces and align normally to the substrates. On the other hand, the exposure of the hydrophobic alkyl tails of PDP to air is preferred because it prevents the high-surface-tension P4VP from contacting air, therefore a PDP brush layer formed at the film-air interfaces. Both of the forces, one from the bottom and the other from the top of the films, drive the P4VP(PDP) lamellae to be parallel to the surface and the films are so thin that the effects can propagate through the entire films. ${ }^{23,26}$

In our previous study, we suggested that the parallel alignment of P4VP(PDP) lamellae plays a crucial role in the formation of perpendicular alignment of PS cylinders in supramolecular thin films under annealing by chloroform. ${ }^{26}$ 
For thin films with sufficiently high grafting density, such as the 1:1 molar ratio of PDP to $4 \mathrm{VP}$ as the present case, the PDP molecules prefer to orient parallel to the long axis of the cylinders to prevent the entropy loss due to the crowding effect of squeezing PDP molecules into the limit space near the nonplanar interfaces of cylinders and thus achieve interface stabilization. ${ }^{47,48}$ As a result, PS cylinders tend to align orthogonally to the P4VP(PDP) lamellae and since the P4VP(PDP) lamellae are parallel to the surface in thin films, PS cylinders are then oriented perpendicular to the surface. As shown in the preceding section, in addition to chloroform, such alignments were also observed in thin films annealed by other nonoxygen-bearing solvents, i.e., toluene and benzene. The mechanism for perpendicular PS cylinders should be the same for all the nonoxygen-bearing solvents. Note that the mechanism during annealing is inferred from the postannealing structural analysis here. Given that the vapor pressures in the annealing setup used in this study are low, the compatibility between PS and P4VP(PDP) can only be slightly enhanced during annealing. The blocks may remain in segregation region such that the proposed alignment can reasonably occur in the swollen films.

Following this argument, the microdomain alignment in thin films annealed by oxygen-bearing solvents is in fact less stable because in such cases PS cylinders and P4VP(PDP) lamellae are both parallel to the surface. It has been shown that the selectivity of solvents to each phase ${ }^{35}$ and the evaporation rates of solvents ${ }^{21}$ may result in varying morphologies and orientation. However, we can find in Table 1 that the solvents in both groups are not particularly selective to PS or P4VP blocks. Furthermore, evaporation rates are generally inversely related to boiling points and the boiling points of the solvents in both groups range from $60^{\circ} \mathrm{C}$ to above $100^{\circ} \mathrm{C}$. The solvents in both groups would not evaporate particularly fast or slowly at a given temperature. Therefore, there should be other factors responsible for the difference in orientation. Since the main difference between these two groups of solvents is whether their chemical structures contain oxygen atoms, we believe that it is such strong electronegative atoms that determine the orientation of PS cylinders in supramolecular thin films, which will be elaborated as follows.

The mechanism of the above proposed orthogonal alignments of PS cylinders and P4VP(PDP) lamellae is based on the assumption that the PDP molecules are grafted onto P4VP. However, the interactions between PDP and P4VP are weak hydrogen bonds whose strengths are subject to change in presence of external stimuli, unlike covalent bonds that are stable in common conditions. During solvent annealing, the solvent molecules diffuse into films and swell the films at a ratio about 1.5 to the original thicknesses. ${ }^{26}$ In other words, there are a significant amount of solvent molecules penetrating films during annealing such that the effects of the solvent molecules on the hydrogen bonds between PDP and P4VP cannot be ignored. In the vapors of nonoxygen-bearing solvents, since the solvent molecules interact with PDP molecules and block copolymers mainly through weak van der Waals interactions, the formation of stronger hydrogen bonds between PDP and $\mathrm{P} 4 \mathrm{VP}$ is enthalpy-favorable for lowering the system free energy. Figure 7a shows the ${ }^{1} \mathrm{H}$ NMR spectra of PDP dissolved in $d$ chloroform and the chemical shift of the hydroxyl hydrogen of PDP is $4.63 \mathrm{ppm}$. When PDP is mixed with PS- $b$-P4VP in a 1:1 molar ratio in chloroform, the peak is shifted to $5.26 \mathrm{ppm}$, confirming the formation of hydrogen bonds between PDP and (a) in d-chloroform

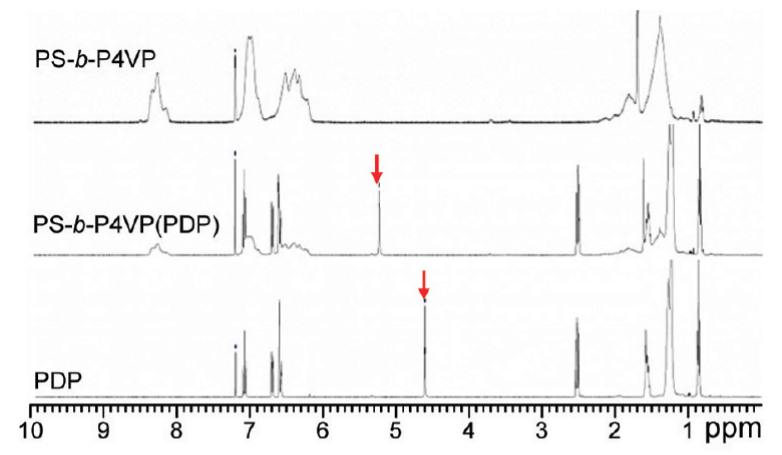

(b) in d-THF

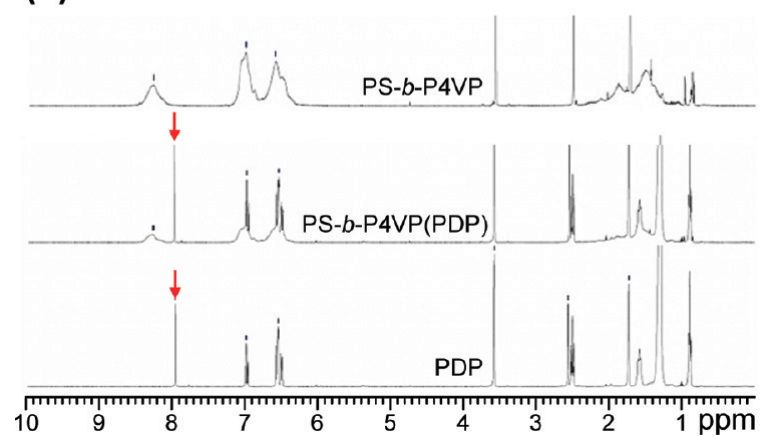

Figure 7. ${ }^{1} \mathrm{H}$ NMR spectra of PDP, PS- $b$-P4VP and PS- $b$-P4VP(PDP) dissolved in (a) $d$-chloroform and (b) $d$-THF. The arrows indicate the chemical shifts of the hydroxyl hydrogen on PDP.

P4VP. ${ }^{49}$ The ${ }^{1} \mathrm{H}$ NMR results are similar for other nonoxygenbearing solvents. The detailed data are listed in Table 2 and the

Table 2. ${ }^{1} \mathrm{H}$ NMR Chemical Shifts of the Hydroxyl Hydrogen on PDP

\begin{tabular}{ccc} 
& \multicolumn{2}{c}{ chemical shift $(\mathrm{ppm})$} \\
\cline { 2 - 3 }$d$-solvent & PDP & PS- $b$-P4VP(PDP $)$ \\
toluene & 3.98 & 4.30 \\
benzene & 4.10 & 4.47 \\
chloroform & 4.63 & 5.26 \\
THF & 7.97 & 7.99 \\
1,4-dioxane & 7.43 & 7.44 \\
DMF & 9.37 & 9.39
\end{tabular}

spectra obtained in toluene and benzene are shown in the Supporting Information, Figures S2 and S3. It is thus suggested that P4VP(PDP) complexes are relatively stable in the vapors of nonoxygen-bearing solvents and PS cylinders prefer to be orthogonal to P4VP(PDP) lamellae to stabilize the interfaces of cylinders.

When thin films are annealed in the vapors of oxygen-bearing solvents, due to the competition of solvent molecules to form hydrogen bonds with PDP molecules through the highly electronegative oxygen atoms, the strength of hydrogen bonds between P4VP and PDP are greatly reduced. In Figure 7b, the chemical shift of hydroxyl hydrogen of PDP alone dissolved in $d$-THF is 7.97 ppm, which is much higher than that of PDP in nonoxygen-bearing solvents. Such a high chemical shift implies that the PDP molecules interact strongly with THF molecules. When PDP and PS- $b$-P4VP are mixed together in $d$-THF, the 
peak position is nearly unchanged, indicating that the interactions between PDP and P4VP are trivial compared to those between PDP and THF molecules. The chemical shifts of the PDP hydroxyl hydrogen in other oxygen-bearing solvents behave in a similar manner, as listed in Table 2 . The spectra obtained in 1,4-dioxane and DMF can be found in the Supporting Information, Figure S4 and S5. The above results suggest that PDP molecules are unable to graft onto P4VP in the presence of oxygen-bearing solvents. During annealing, PDP molecules are relatively free so that the crowding effect near the interface of PS cylinders can be avoided and the driving force for inducing perpendicular PS cylinders is absent. Instead, the preferential interaction between P4VP and silicon substrate attracts P4VP chains to the substrate and in turn, PS cylinders are drawn to be parallel. Note that although PDP molecules are unbound during annealing, from the GISAXS data we can still see order parallel P4VP(PDP) lamellae after annealing. It is possible that most PDP molecules are still framed in their originally located P4VP domains so that they can be rapidly associated with P4VP upon solvent evaporation when films are removed from the annealing setup.

The interplays among PDP, P4VP, and solvent molecules mentioned above can be verified by the simple solubility tests of P4VP in the solvents. P4VP homopolymer with $M_{\mathrm{w}} \sim 60$ $000 \mathrm{~g} / \mathrm{mol}$ is insoluble in toluene, benzene, and 1,4-dioxane and only slightly swollen in THF. For nonoxygen-bearing solvents, i.e. toluene and benzene, after the addition of equimolar PDP into P4VP homopolymer solutions, P4VP becomes soluble. On the other hand, the addition of PDP is unable to enhance the solubility of P4VP homopolymer in THF and 1,4-dioxane. The results confirm that PDP molecules are hydrogen-bonded with P4VP in nonoxygen-bearing solvents and work as a compatibilizer between $\mathrm{P} 4 \mathrm{VP}$ and the solvents, while such hydrogen bonds are missing in oxygen-bearing solvents. On the basis of what reveals here, we would like to point out the importance of the solvents used in preparing supramolecules. Supramolecules are formed through secondary bonds and the strengths of such weak interactions will be significantly affected by the types of solvents where the supramolecules are supposed to be formed. If solvents are not properly selected, the building components of supramolecules may not be successfully associated, even though each individual component can be well-dissolved. One example is P4VP and PDP mixed in DMF, where both P4VP and PDP can dissolve but the interaction between P4VP and PDP would be very weak.

\section{CONCLUSIONS}

We have demonstrated that the process of solvent annealing would greatly affect the microdomain orientation in thin films formed by PS- $b$-P4VP(PDP) supramolecules. When films are annealed by nonoxygen-bearing solvents, including toluene, benzene and chloroform, PS cylinders are perpendicular to the surface. This is due to the tendency of PS cylinders to be orthogonal to the parallel P4VP(PDP) lamellae at high molar ratio of $\mathrm{PDP}$ to $4 \mathrm{VP}$ unit and such an preferential alignment is based on the fact that PDP molecules graft onto P4VP through hydrogen bonds in the vapors of the nonoxygen-bearing solvents. PS cylinders, however, are parallel to the surface when films are annealed by oxygen-bearing solvents, such as THF, 1,4-dioxane, and DMF. From the ${ }^{1} \mathrm{H}$ NMR results, we found the PDP molecules interact strongly with the oxygen-bearing solvent molecules due to the electronegative nature of oxygen atom, while the interactions between PDP and P4VP become very weak. Such weak interactions are insufficient to lead to the formation of $\mathrm{P} 4 \mathrm{VP}(\mathrm{PDP})$ complexes in oxygen-bearing solvents. As a result, the driving force for perpendicular PS cylinders disappears and the cylinders remain parallel. This work provides a simple and highly efficient approach to manipulate the microdomain orientation in block copolymer thin films, and it also emphasizes the advantage of the reversibility of supramolecules.

\section{ASSOCIATED CONTENT}

\section{S Supporting Information}

AFM images of thin films annealed by other solvents and ${ }^{1} \mathrm{H}$ NMR spectra obtained in deuterated toluene, benzene, 1,4dioxane, and DMF. This material is available free of charge via the Internet at http://pubs.acs.org/.

\section{AUTHOR INFORMATION}

\section{Corresponding Author}

*E-mail: shtung@ntu.edu.tw.

\section{ACKNOWLEDGMENTS}

This work was financially supported by the Taiwan National Science Council (NSC 99-2221-E-002-021). We acknowledge NSRRC, Taiwan, for facilitating the GISAXS experiments performed as part of this work and Dr. U-Ser Jeng, Dr. ChunJen $\mathrm{Su}$, and Dr. Chiu-Hun Su of NSRRC for the assistance in scattering experiments.

\section{REFERENCES}

(1) Bates, F. S.; Fredrickson, G. H. Annu. Rev. Phys. Chem. 1990, 41, $525-557$.

(2) Hamley, I. W. Prog. Polym. Sci. 2009, 34 (11), 1161-1210.

(3) Kim, S. O.; Solak, H. H.; Stoykovich, M. P.; Ferrier, N. J.; de Pablo, J. J.; Nealey, P. F. Nature 2003, 424 (6947), 411-414.

(4) van Zoelen, W.; ten Brinke, G. Soft Matter 2009, 5 (8), 15681582.

(5) Black, C. T. ACS Nano 2007, 1 (3), 147-150.

(6) Black, C. T.; Ruiz, R.; Breyta, G.; Cheng, J. Y.; Colburn, M. E.; Guarini, K. W.; Kim, H. C.; Zhang, Y. IBM J. Res. Dev. 2007, 51 (5), 605-633.

(7) Park, S.; Lee, D. H.; Xu, J.; Kim, B.; Hong, S. W.; Jeong, U.; Xu, T.; Russell, T. P. Science 2009, 323 (5917), 1030-1033.

(8) Ryu, D. Y.; Shin, K.; Drockenmuller, E.; Hawker, C. J.; Russell, T. P. Science 2005, 308 (5719), 236-239.

(9) Huang, E.; Rockford, L.; Russell, T. P.; Hawker, C. J. Nature 1998, 395 (6704), 757-758.

(10) Fasolka, M. J.; Mayes, A. M. Annu. Rev. Mater. Res. 2001, 31, 323-355.

(11) Han, E.; Stuen, K. O.; Leolukman, M.; Liu, C. C.; Nealey, P. F.; Gopalan, P. Macromolecules 2009, 42 (13), 4896-4901.

(12) Ji, S.; Liu, C. C.; Son, J. G.; Gotrik, K.; Craig, G. S. W.; Gopalan, P.; Himpsel, F. J.; Char, K.; Nealey, P. F. Macromolecules 2008, 41 (23), 9098-9103.

(13) Segalman, R. A.; Yokoyama, H.; Kramer, E. J. Adv. Mater. 2001, 13 (15), 1152-1155.

(14) Bita, I.; Yang, J. K. W.; Jung, Y. S.; Ross, C. A.; Thomas, E. L.; Berggren, K. K. Science 2008, 321 (5891), 939-943.

(15) Tang, C. B.; Lennon, E. M.; Fredrickson, G. H.; Kramer, E. J.; Hawker, C. J. Science 2008, 322 (5900), 429-432.

(16) Xu, T.; Zvelindovsky, A. V.; Sevink, G. J. A.; Lyakhova, K. S.; Jinnai, H.; Russell, T. P. Macromolecules 2005, 38 (26), 10788-10798.

(17) Villar, M. A.; Rueda, D. R.; Ania, F.; Thomas, E. L. Polymer 2002, 43 (19), 5139-5145.

(18) Lin, Z. Q.; Kim, D. H.; Wu, X. D.; Boosahda, L.; Stone, D.; LaRose, L.; Russell, T. P. Adv. Mater. 2002, 14 (19), 1373-1376. 
(19) Ho, R. M.; Tseng, W. H.; Fan, H. W.; Chiang, Y. W.; Lin, C. C.;

Ko, B. T.; Huang, B. H. Polymer 2005, 46 (22), 9362-9377.

(20) Park, S.; Wang, J. Y.; Kim, B.; Xu, J.; Russell, T. P. ACS Nano

2008, 2 (4), 766-772.

(21) Phillip, W. A.; Hillmyer, M. A.; Cussler, E. L. Macromolecules

2010, 43 (18), 7763-7770.

(22) Sidorenko, A.; Tokarev, I.; Minko, S.; Stamm, M. J. Am. Chem. Soc. 2003, 125 (40), 12211-12216.

(23) Albrecht, K.; Mourran, A.; Zhu, X.; Markkula, T.; Groll, J.; Beginn, U.; deJeu, W. H.; Moeller, M. Macromolecules 2008, 41 (5), $1728-1738$.

(24) Tung, S. H.; Kalarickal, N. C.; Mays, J. W.; Xu, T. Macromolecules 2008, 41 (17), 6453-6462.

(25) Kao, J.; Tingsanchali, J.; Xu, T. Macromolecules 2011, 44 (11), $4392-4400$.

(26) Lee, C.-H.; Tung, S.-H. Soft Matter 2011, 7 (12), 5660-5668.

(27) Lee, J. W.; Lee, C.; Choi, S. Y.; Kim, S. H. Macromolecules 2010, 43 (1), 442-447.

(28) Ruokolainen, J.; Saariaho, M.; Ikkala, O.; ten Brinke, G.; Thomas, E. L.; Torkkeli, M.; Serimaa, R. Macromolecules 1999, 32 (4), $1152-1158$.

(29) Ikkala, O.; ten Brinke, G. Science 2002, 295 (5564), 2407-2409.

(30) Ikkala, O.; ten Brinke, G. Chem. Commun. 2004, 19, 2131-2137.

(31) Valkama, S.; Ruotsalainen, T.; Nykanen, A.; Laiho, A.; Kosonen,

H.; ten Brinke, G.; Ikkala, O.; Ruokolainen, J. Macromolecules 2006, 39 (26), 9327-9336.

(32) Park, S.; Kim, B.; Xu, J.; Hofmann, T.; Ocko, B. M.; Russell, T. P. Macromolecules 2009, 42 (4), 1278-1284.

(33) Xuan, Y.; Peng, J.; Cui, L.; Wang, H. F.; Li, B. Y.; Han, Y. C. Macromolecules 2004, 37 (19), 7301-7307.

(34) Elbs, H.; Drummer, C.; Abetz, V.; Krausch, G. Macromolecules 2002, 35 (14), 5570-5577.

(35) Bosworth, J. K.; Paik, M. Y.; Ruiz, R.; Schwartz, E. L.; Huang, J. Q.; Ko, A. W.; Smilgies, D.-M.; Black, C. T.; Ober, C. K. ACS Nano 2008, 2 (7), 1396-1402.

(36) Tokarev, I.; Krenek, R.; Burkov, Y.; Schmeisser, D.; Sidorenko, A.; Minko, S.; Stamm, M. Macromolecules 2005, 38 (2), 507-516.

(37) Nandan, B.; Vyas, M. K.; Bohme, M.; Stamm, M. Macromolecules 2010, 43 (5), 2463-2473.

(38) Cordeiro, J. M. M.; Freitas, L. C. G. Z. Naturforsch., Sect. a-a J. Phys. Sci. 1999, 54 (2), 110-116.

(39) Lim, W. K.; Rosgen, J.; Englander, S. W. Proc. Natl. Acad. Sci.

U.S.A. 2009, 106 (8), 2595-2600.

(40) Tung, S. H.; Xu, T. Macromolecules 2009, 42 (15), 5761-5765.

(41) van Zoelen, W.; Asumaa, T.; Ruokolainen, J.; Ikkala, O.; ten Brinke, G. Macromolecules 2008, 41 (9), 3199-3208.

(42) van Zoelen, W.; Polushkin, E.; ten Brinke, G. Macromolecules 2008, 41 (22), 8807-8814.

(43) Jeng, U. S.; Su, C. H.; Su, C. J.; Liao, K. F.; Chuang, W. T.; Lai, Y. H.; Chang, J. W.; Chen, Y. J.; Huang, Y. S.; Lee, M. T.; Yu, K. L.; Lin, J. M.; Liu, D. G.; Chang, C. F.; Liu, C. Y.; Chang, C. H.; Liang, K. S. J. Appl. Crystallogr. 2010, 43, 110-121.

(44) Brandrup, J.; Immergut, E. H.; Grulke, E. A.; Abe, A.; Bloch, D. R. Polymer Handbook, 4th ed.; John Wiley \& Sons: New York, 2005.

(45) Matsen, M. W. J. Chem. Phys. 1997, 106 (18), 7781-7791.

(46) Lee, B.; Park, I.; Yoon, J.; Park, S.; Kim, J.; Kim, K. W.; Chang,

T.; Ree, M. Macromolecules 2005, 38 (10), 4311-4323.

(47) Osuji, C.; Zhang, Y. M.; Mao, G. P.; Ober, C. K.; Thomas, E. L. Macromolecules 1999, 32 (22), 7703-7706.

(48) Verploegen, E.; McAfee, L. C.; Tian, L.; Verploegen, D.; Hammond, P. T. Macromolecules 2007, 40 (4), 777-780.

(49) Wagner, G.; Pardi, A.; Wuthrich, K. J. Am. Chem. Soc. 1983, 105 (18), 5948-5949. 